\title{
In vitro reproduction of Kidney Tea (Orthosiphon
}

\section{stamineus Bents)}

Nana Zarnadze, Inga Diasamidze, Natela Varshanidze, Ketevan Dolidze and Tsiala Bolkvadze

Shota Rustaveli State University, Batumi 6010, Georgia

\begin{abstract}
The stages of introduction in vitro culture of the local population of the kidney tea (Orthosiphon stamineus Bents) cultivated in the Georgia's medicinal plant farm and the microclonal propagation, in particular, have been elaborated. The cultivation of explants was carried out on the Gamborg (B5) feeding area. The hormonal (BAP; Zn; NAA) composition of the feeding area and their concentrations have been selected; proliferation of buds in the basal part of the sprout has been achieved from the formed morphogenic tissue. The microclones by activating axillary meristem have been received.
\end{abstract}

Key words: Kidney tea, in vitro culture, reproduction, plant regeneration, micropropagation, phytohormones, adventive buds.

\section{Introduction}

High ability to regenerate plant and cell in vitro culture makes it possible to maximize the ability of getting full-fledged plants from somatic tissue. The proliferation induction of the axillary meristems of the isolated organs is connected to the hormonal balance of different plant organs, the transportation of phytohormones and to the supply of buds with trophic factors [1]. During the insufficient restoration of the organs by the isolated parts and the regeneration of the perfect plant, the correlation among the whole plant organs is violated and at the same time this correlation is compensated, which results in the compensation of the balance in the organism. This is the most common method of regeneration in higher plants, based on the methods of vegetative, as traditional propagation as well as on the methods of cell and tissue culture [2, 3]. These processes are regulated by phytohormones. The selection of concentration of phytohormones in the feeding area is carried out by visual observation on the reaction of the explants based on the trial and error. However, we should note that there is no ideal formula for the type and concentration of phytohormones in the

Corresponding author: Nana Zarnadze, doctor of biology, assoc. prof., research fields: development biology. feeding area.

The aim of our experiment was to obtain sterile cultures of local population of kidney tea (Orthosiphon stamineus Bents) (Fig. 1) cultivated on the farm of medicinal plants of Georgia (Kobuleti); to develop and optimize microclonic propagation conditions. As known from the scientific literature, various experiments have been conducted by various scientists, around Orthosiphon stamineus in vitro cultivation; the efficacy of micropropagation in these works has been achieved by the activation of the meristematic tissue of buds and the induction and regeneration of the callusing on the MS feeding area with various hormonal composition [4-6].

The kidney tea brought into culture in the subtropical regions of Georgia is a one-year culture, strongly branched evergreen bush with a height of $70 \mathrm{~cm}$. The root system is brush-like and strongly branched. The stems are square; the leaves are short-stalked and oppositely arranged. The leaves in the lower part of the stem are downy along the vessels, the flowers are mild-violet, androgynous. The scorpioid creates pyramidal cluster; the fruit consists of 1-4 seeds; blossoms in July-September. Leaves and sprouts used for treatment contain triterpenoid saponins, flavonoids, fustic substances, ether oil, lipids, organic acids: wine, 
lemon phenolcabon, and rosemary acids. Kidney tea is mainly used as a diuretic mean for acute and chronic diseases of kidneys, including cystitis, urethritis, diabetes, cholecystitis, gallbladder disease, heart diseases, which are accompanied by swelling. It removes the urea, uric acid and chlorides from the organism. Taking renal tea is very effective and it does not have contraindications.

Due to the above features, getting the seedlings of kidney tea is very important for plant experimental stations and farms. The need for seedling material is great enough to ensure the traditional methods of multiplication, so this problem was solved by developing the tissue culture technology.

\section{Methodology and Materials}

\subsection{Experiment Design}

In order to get sterile crops, as a sterilizing substance, we studied and examined the $0.2 \%$ water solution of "Diocide" (composition: Ethilmercuric chloride, Cetylpyridinium chloride). As primary materials, we used kidney tea seeds, which were rinsed with soap and running water and left under water pressure for 0.5-1.0 hours, and then rinsed again with distilled water several times. We carried the rest of the manipulations in aseptic conditions - in laminar boxes. In the beginning, we were placing the explants in sterilizing solution for 15 minutes (Diocide). Then we washed them with sterilized water several times (3-4 times) and left them in the last portion of water for 10-15 minutes. After completion of sterilization, we were able to sow the explants on the feeding area made according to Gamborg (B5) formula [7], which was added by $0.7 \%$ $(\mathrm{w} / \mathrm{v})$ Agar (agar-agar, sigma), 3\% (w/v) sucrose, $\mathrm{pH}=$ 5, 6-5.8; The food was sterilized under 0.9-1 \pm 0.1 atmospheric pressure, for 20 minutes. The incubation of cultures was carried out on light (illumination 3 kilolux, 16/8 hours photoperiod, temperature $-25 \pm$ $1{ }^{\circ} \mathrm{C}$, relative humidity of air $70 \%$ ). At the microclonic propagation phase, we used the buds of in vitro cultivated crops.
From the growth regulators we have learnt the joint action of various concentrations of cytokines (BAP and Zeatin) and Auxin (NAA) on the induction and proliferation of buds.

\subsection{Statistical Analysis}

We carried out the recording of experimental results in every 25-30 days, within 10-12 months. The biological repetition of each test variant constituted 100 units.

When studying any organogenesis, we counted the number of initials on the sown explants and expressed with average arithmetic. The data on the number of shoots obtained through bud proliferations shoot and node number per explants and shoot length were analyzed using ANOVA. The student's $t$-test was applied at $p=0.05$ probability level to find significant differences between the means.

\section{Results and Discussion}

The results of sterilization showed that the used sterilent was effective for getting aseptic material. And $0.2 \%$ Diocide water solution with the exposition provided 15 minutes for sterilization of seeds by $97 \%$, and $93 \%$ of sterile seeds were with the ability to grow. Seeds started to grow in 6-8 days after sowing and in 20 days we have in vitro well-growing cultures.

At microclonal propagation stage, the increase in the propagation coefficient was indicated by cytokinins influence; in the process of 0 and I subcultivation the influence of hormones was going on with a different effect; and both studied cytokinins resulted in the introduction and development of new axillary buds on the main explant. Explants have shown a high level of morphogenetic potential from 0-subcultivation. Bud initiation was observed in 5-7 days after sowing, and in 9-10 days-intensive growth. In the second half of the subcultivation-from the 15th day, the renal tea cultures have been well-developed explants.

The results of the experiment demonstrated that the propagation coefficient indicator, the form and 
character of the growth of the buds and the sprouts, the activation of the axillary meristem and the formation of additional adventive bud depended on the use of cytokinins and their concentration. Both BAP and Zeatin reacted positively on the process of micro-propagation. Both hormones caused stem morphogenesis: adventive bud-formation and the activation of axillary meristems.

Among the experienced cytokinins, BAP was the most effective. At the end of 0 and I subcultivation the main sprout height was $95 \mathrm{~mm}$ on the feeding area containing $5 \mathrm{mkm}$ BAP, and the average number of the interarticulars of the mother bud was 9.5 units (Table 1). Some micro sprouts, along with an apical growth, developed 2-3 additional buds in the basal part. The concentration of $10 \mathrm{mkm}$ resulted in the initiation of axillary meristems and induction of lateral buds. Near the basal part of the main stem, the buds from two parallel axillary meristems were developed simultaneously, mostly the development of the first two lower buds were accompanied by the initiation of the growth of the third and fourth axillary buds. This process was more intensive in the feeding area with the combination of BAP and NAA than in the feeding area containing only BAP.

BAP concentration increase $(15 \mathrm{mkm})$ in the feeding area (Table 1) caused the shortening of the main sprout $(78.8 \mathrm{~mm})$, that resulted in reducing the number of interarticulars (6.8), but instead, several buds developed instead of one bud from the axillary meristems of the interarticulars (Fig. 2). Presumably, the concentration of hormones was going on in the meristem base of the axillary bud that supported the initiation of the primordial buds and initiating their growth. The rest of the remaining axillary meristems came out of the state of rest after the removal of regenerated buds and moving to the new feeding area (Fig. 3).

Twenty mkm concentration of BAP has contributed to a significant reduction in the number of interarticulars and the massive formation of the adventive buds in the hypocotyl part of the explant. They are the secondary explants for the next cycle of propagation (Fig. 4).

Zeatin exercised similar morphogenetic processes relatively less intensely than BAP. There have often been several simultaneous developments of several apically growing sprouts, among which it was difficult to distinguish the main one. In our opinion, the action of Zeatin was due to the fact that kidney tea is a herbaceous plant; the genotype is quite mobile and its ability to fully detect morphogenetic potential is the effect of Zeatin, i.e., Zeatin, by its gentle exposure, allows the plant to realize its ability of totipotency. The plant-microclones developed on the feeding area containing Zeatin completely repeated the morphology of the mother-plant, and the buds developed on the feeding area containing the high concentrations of BAP

Table 1 Influence of hormones on the intensity of proliferation of micro-sprouts of renal tea $\boldsymbol{n}=\mathbf{1 0 0}$.

\begin{tabular}{|c|c|c|c|c|c|}
\hline \multicolumn{3}{|c|}{$\begin{array}{l}\text { Hormones } \\
(\mathrm{mkm})\end{array}$} & \multirow[t]{2}{*}{$\begin{array}{l}\text { The average length of the } \\
\text { micro-sprouts (mm) }\end{array}$} & \multirow[t]{2}{*}{$\begin{array}{l}\text { The total number of } \\
\text { interarticulars }\end{array}$} & \multirow[t]{2}{*}{$\begin{array}{l}\text { Average number of adventive } \\
\text { buds }\end{array}$} \\
\hline $\mathrm{Zn}$ & BAP & NAA & & & \\
\hline 5 & & & $92.2 \pm 0.21$ & $8.2 \pm 0.11$ & $2.3 \pm 0.50$ \\
\hline 10 & & & $80.0 \pm 0.32$ & $6.9 \pm 0.44$ & $22.4 \pm 0.22$ \\
\hline 15 & & & $38.5 \pm 0.38$ & $5.0 \pm 0.4$ & $36.0 \pm 0.18$ \\
\hline \multirow[t]{8}{*}{20} & & & $23.4 \pm 0.30$ & $4.0 \pm 0.4$ & $50.1 \pm 0.11$ \\
\hline & 5 & & $95.1 \pm 0.25$ & $9.5 \pm 0.3$ & $3.1 \pm 0.54$ \\
\hline & 10 & & $86.8 \pm 0.37$ & $7.1 \pm 0.4$ & $38.9 \pm 0.13$ \\
\hline & 15 & & $78.8 \pm 0.36$ & $6.0 \pm 0.4$ & $44.9 \pm 0.32$ \\
\hline & 20 & & $41.0 \pm 0.28$ & $4.0 \pm 0.5$ & $52.0 \pm 0.12$ \\
\hline & 10 & 1 & $75.3 \pm 0.35$ & $6.5 \pm 0.32$ & $50.1 \pm 0.11$ \\
\hline & 10 & 2 & $64.9 \pm 0.48$ & $5.9 \pm 0.4$ & $47.5 \pm 0.11$ \\
\hline & 10 & 3 & $52.7 \pm 0.46$ & $4.8 \pm 0.4$ & $39.3 \pm 0.21$ \\
\hline
\end{tabular}




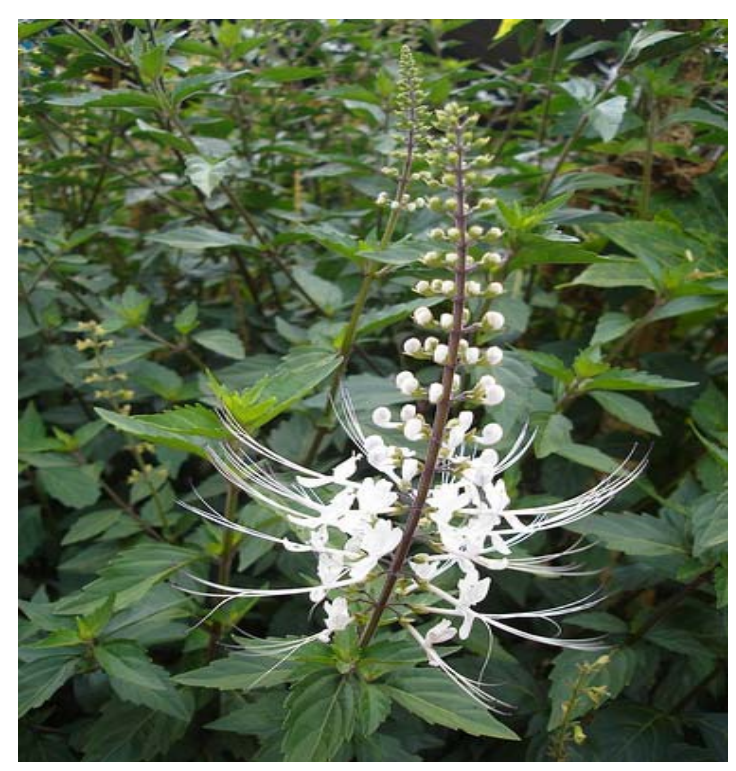

Fig. 1 Kidney tea.

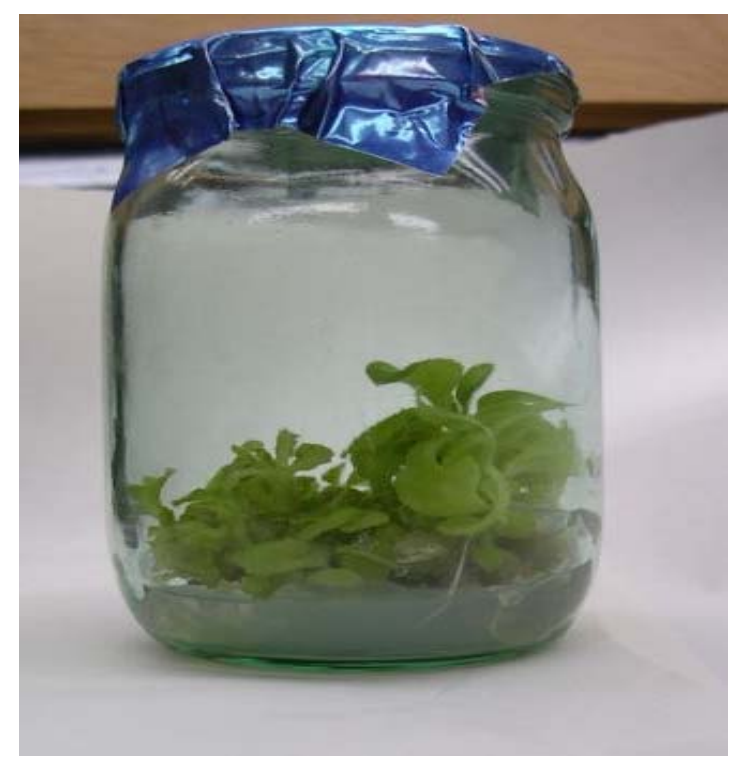

Fig. 2 Development shoot on Gamborg medium with 15 mkm BAP.

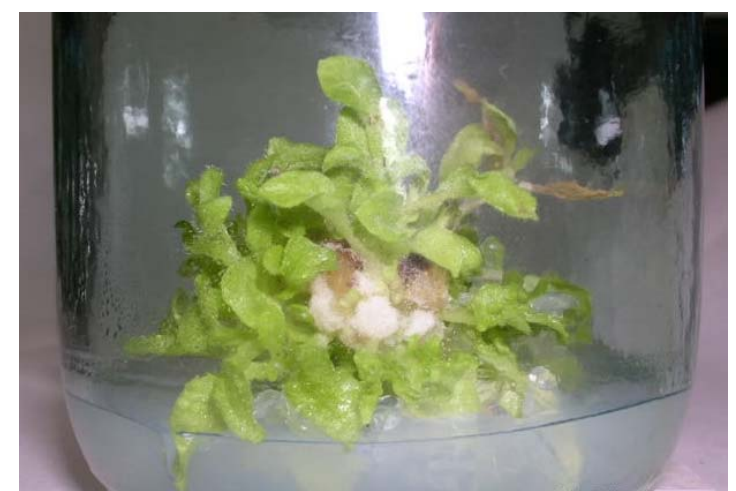

Fig. 3 Adventive bud-formation on Gamborg medium with 15 mkm BAP + 3 mkm NAA.

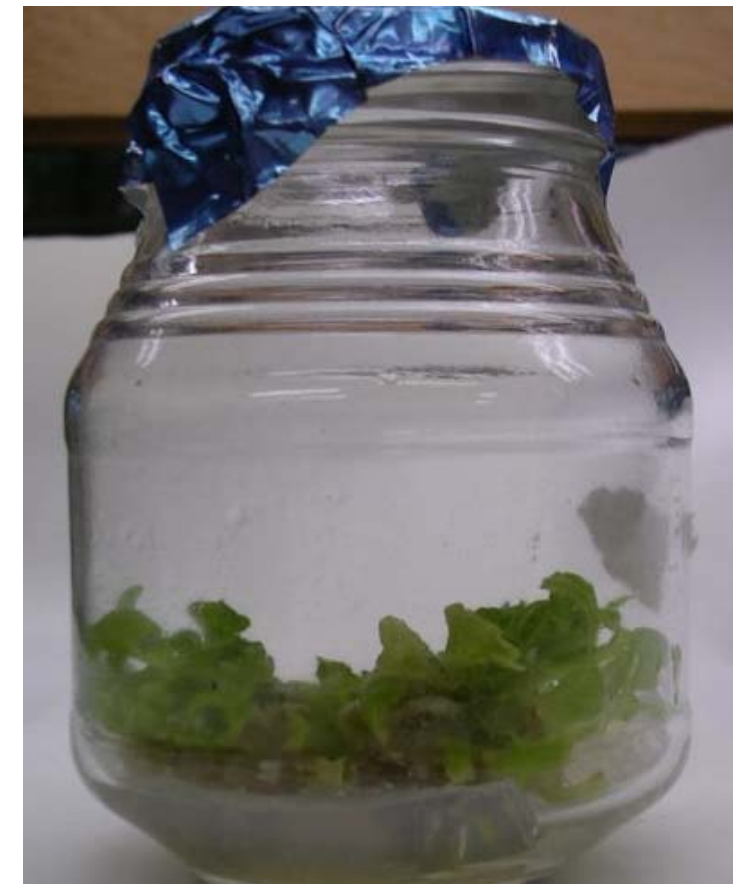

Fig. 4 The massive formation of the adventive buds on Gamborg medium with 20 mkm BAP.

were insignificantly but morphologically different (in the form of a leaf plate). Although Zeatin as a hormone was quite effective, sprouts needed more time to develop than BAP. The optimum concentration of the Zeatin was $15 \mathrm{mkm}$ and the indicator for Benzylaminopurine was $10 \mathrm{mkm}$.

In the experiment we studied the influence of NAA with cytokinins, namely with BAP on the formation of buds. As shown in Table 1, the adventive bud formation in renal tea culture has been stimulated by low concentrations of NAA in the feeding area with BAP. It facilitated the formation of morphogenetic callus in the basal part of the explant. This process was going on intensively by increasing the concentration of NAA (1-3 mkm). The morphogenetic potential of the callus was intensifying and the micro-propagation coefficient was growing. The increase in concentrations of NAA had been ineffective, since there was a massive formation of callus and multiplication coefficient was decreased.

The experimental research has shown us the ability to successfully use the in vitro method to propagate kidney tea. 


\section{Conclusions}

The experimental research has shown us the ability to successfully use the in vitro method for massive transplantation of the kidney tea (Orthosiphon stamineus Bents).

For the receiving uninfected and viable cultures, it is best to use $0.2 \%$ of the diocide solution by exposing 15 min.

For the efficiency of kidney tea microclonic multiplication, it is optimal to provide trophic provision of micro-grafts with the modified Gamborg food area.

For increasing the kidney tea multiplication coefficient in vitro culture has given us the best results of $10 \mathrm{mkm}$ of BAP and $15 \mathrm{mg}$ concentrations of Zeatine. In order to stimulate the micropropagation process, in addition to cytokines, a small amount of hormone ( $3 \mathrm{mkm}$ ) of the Auxin nature (NAA) is added to the food area.

Making up the protocol of kidney tea micropropagation will enable us to successfully use this method in the future for the cultivation of healthy plants in genetically-selective works of medicinal plants.

\section{References}

[1] Kefeli V. I. 1978. "Plant Growth and Natural Regulators." Physiology of the Plant 5: 975-89.

[2] Lomtatidze, N., Zarnadze, N., Alasania, N., and Zarnadze, R. 2009. "In Vitro Morphogenesis of Loquat (Eriobotria japonica L.)." Bulletion of the Georgian National Academy of Sciences 3 (1).

[3] Zarnadze, N., Manjgaladze, S., and Bolkvadze, T. S. 2015. "Peculiarities of Microclonal Propagation of the Plants." Presented at the International Conference Applied Ecology: Problems, Innovations, 7-10 May, 2015, Tbilisi-Batumi, Georgia.

[4] Sheena, E. V., and Jothi, G. Y. 2015. "In Vitro Propagation of Orthosiphon stamineus Benth (Lamiaceae) an Important Medicinal Plant Using Nodal and Leaf Explants.” The Pharma Innovation Journal 4 (7): 06-10.

[5] Mohd Nawi, I. H., and Abd Samad, A. 2012. "Successful Plant Regeneration of Orthosiphon stamineus from Petiole.” Journal of Medicinal Plants Research 6 (26): 4276-80.

[6] Lee, W. L., and Chan, L. K. 2004. "Plant Regeneration from Stem Nodal Segments of Orthosiphon stamineus Benth., a Medicinal Plant with Diuretic Activity.” In Vitro Cell. Dev. B 40: 115-8.

[7] Gamborg, O. L., Miller, R. A., and Ojima, K. 1968. "Nutrient Requirements of Suspension Cultures of Soybean Root Cells.” Exp. Cell Res. 50: 151-8. 